\title{
Effects of blockage of the impeller channel on performances of non-overload centrifugal pump
}

\author{
Wanfang Zhao ${ }^{1, a}$ \\ ${ }^{1}$ Tianjin Maritime College, Tianjin, 300350, China. \\ aEE-mail: tjzwf_001@163.com.
}

Keywords: Centrifugal pump; Blockage; Numerical research.

\begin{abstract}
The shaft power curve of low specific-speed centrifugal pump is a steep rise with the increase of flow rate. The blockage of the impeller channel can reduce effectively the shaft power of low specific-speed centrifugal pump, and achieve pump's non-overload characteristics. In this paper, the flow channel of impeller was blocked three times, the characteristic curves of the four Plans were analyzed comparatively, the turbulent kinetic energy distribution of impeller channel was researched, and the change reasons of centrifugal pump's performance curves were further revealed. The results showed that blockage of the impeller channel can decrease shaft power, and to give reference for design of non-overload centrifugal pump.
\end{abstract}

\section{Introduction}

At low flow rate, low specific-speed centrifugal pump have greater impact loss and the pump's $H-Q$ curve is easy to produce hump. In addition, pump's $P_{\text {shaft }}-Q$ curve grows increasingly steep as flow rate is increased[1]. Some agricultural pumps and industrial pumps have the characteristics such as the operating conditions with several variable and without flow regulating valve on the discharge pipe, motor burned accident due to overload is often happened[2]。

The essence of the impeller channel's blockage is decreasing the impeller flow area, which can reduce shaft power of pump and increase the stability of $H-Q$ curve. In this paper, the flow channel of impeller was blocked three times, using Pumplinx software to predict centrifugal pump performance, and was compared with the prototype pump. The aim was to find a better impeller blocking scheme, to explore the internal cause of effects of impeller's blockage on performances of non-overload centrifugal pump. Therefore, it is essential to focus on effects of impeller's blockage Plans on pump's performances.

\section{Pump main geometric parameters}

This research focuses on the investigation of the low specific-speed non-overload centrifugal pump. All pumps' rotational speed is $2900 \mathrm{r} / \mathrm{min}$. Table 1 lists the main geometric parameters of the prototype pump.

Table 1. The main geometric parameters of the prototype pump.

\begin{tabular}{|c|c|c|}
\hline Nominal geometric parameters & Parameter & Value \\
\hline volume flow rate $\left(\mathrm{m}^{3} / \mathrm{h}\right)$ & $Q_{\mathrm{s}}$ & 15 \\
\hline Head $(\mathrm{m})$ & $H$ & 32 \\
\hline Impeller inlet diameter $(\mathrm{mm})$ & $D_{1}$ & 58 \\
\hline Impeller discharge diameter(mm) & $D_{2}$ & 170 \\
\hline Impeller outlet width $(\mathrm{mm})$ & $b$ & 6 \\
\hline${\text { Blade inlet angle }\left({ }^{\circ}\right)}^{\circ}$ & $\beta_{1}$ & 25.8 \\
\left.\hline${\text { Blade outlet angle }\left({ }^{\circ}\right)}\right)$ & $\beta_{2}$ & 14 \\
\hline Numbers of blade & $Z$ & 4 \\
\hline Rotational speed $(\mathrm{r} / \mathrm{min})$ & $n$ & 2900 \\
\hline
\end{tabular}




\section{Numerical investigation}

PumpLinx is a kind of CFD software based on Cartesian grid of CAB algorithm. It uses the SIMPLE (Semi-Implicit Method for Pressure Linked Equations) algorithm, and turbulence model is standard $k-\varepsilon$ model[3]. There are the advantages of rapid convergence rate, adaptive good and high precision. Fig. 1 shows Cartesian grid of impeller channels of different Plans.
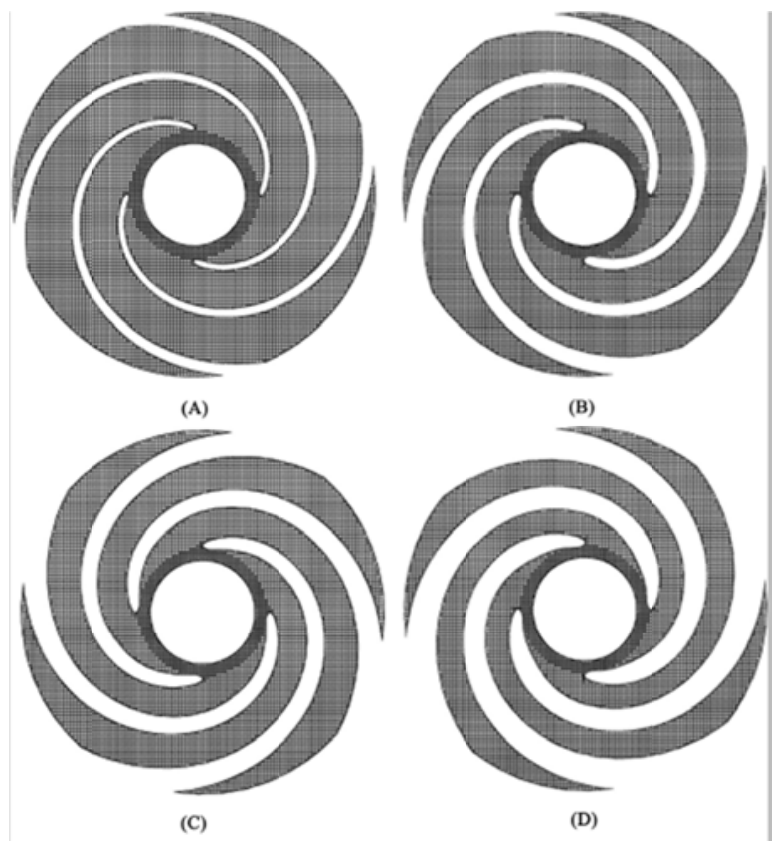

Fig. 1. Generated Cartesian grid for impeller channels of different Plans

\section{Analysis of mesh number}

Independence of mesh number with prototype pump was researched. Because the efficiency was the comprehensive embodiment of pump performances, so the hydraulic efficiency was selected as the independent indicators to verify independence of mesh. When the mesh number was above 0.25 million, the variation of efficiency was less than $0.5 \%$ as shown in Fig. 2. Therefore, the total number of mesh for numerical calculation was more than 0.25 million in all pump models.

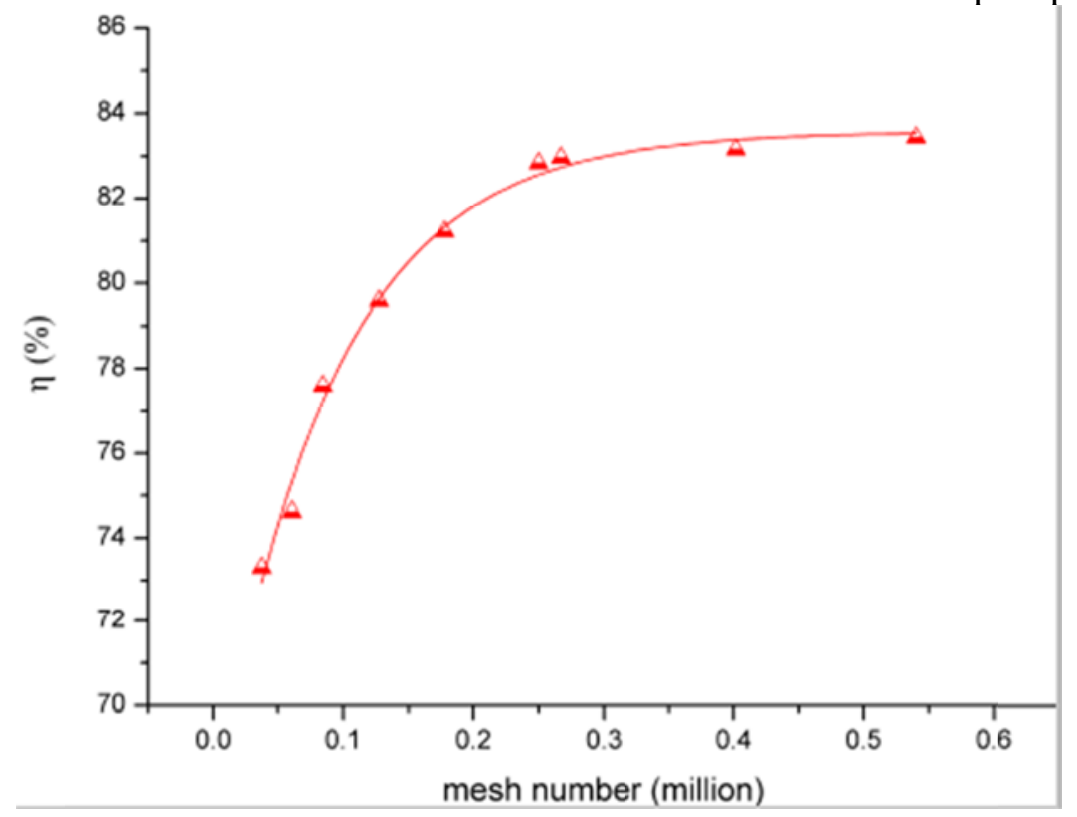

Fig. 2. Pump's efficiency and mesh number curve. 


\section{Solution parameters and convergence discrimination}

The convergence criterion was $10^{-6}$. The clear water at $25^{\circ} \mathrm{C}$ was selected as the fluid. The wall surface was set smooth. The boundary conditions were set to static pressure inlet and volume flow rate outlet. The centrifugal pump's performance curves were obtained by changing the volume flow rate.

\section{Research Plans}

Overload for non-overload centrifugal pump will not occur within the total head operation, Centrifugal pump's maximum shaft power was estimated by the following formula.

$$
\begin{gathered}
P_{\max }=\frac{\rho}{4 \eta_{m}} K_{3} u_{2}^{3} \pi D_{2} b_{2} \varphi_{2} h_{0}^{2} \operatorname{tg} \beta_{2} \\
h_{0}=\frac{2 v_{m 2}}{u_{2} \operatorname{tg} \beta_{2}}
\end{gathered}
$$

$u_{2}$ is circumferential velocity of the blade outlet, $\mathrm{m} / ; \varphi_{2}$ is excretion coefficient of the blade outlet; $\eta_{m}$ is the efficiency of disk friction loss of centrifugal pump, $\% ; K_{3}$ is the correction coefficient; $v_{m 2}$ is the meridional velocity of blade outlet.

$\mathrm{Y}$ was area ratio that was calculated by the formula 3, which was a major determinant of centrifugal pump's head, flow and shaft power. If $\mathrm{Y}<1.0$, the pump efficiency may be lower; If $\mathrm{Y}>$ 2.0, the pump shaft power curve may be overloaded. $F_{t}$ is throat area, $\mathrm{mm}^{2}$.

$$
Y=\frac{\pi D_{2} b_{2} \varphi_{2} \sin \beta_{2}}{F_{t}}
$$

Therefore, decreasing $\varphi_{2}$ can control $P_{\max }$ and the impeller outlet area $F_{2}$, which was beneficial to realize overload characteristics of pump. The reduction of $\varphi_{2}$ can be done by blocking impeller passage. Therefore, this article adopted the method of blocking impeller passage to improve the hydraulic performance of pump.

The Plan of blockage of the impeller channel can reduce $\varphi_{2}$, which can reduce the shaft power of pump. This paper used four kinds of Plans, Table 2 listed the main geometric parameters of the prototype pump. $F_{2} / F_{1}$ was the area ratio of the export and import's effective part between the two blades.

Table 2. Comparison of impeller design parameters among different Plans.

\begin{tabular}{|c|c|c|c|c|}
\hline & Plan A & Plan B & Plan C & Plan D \\
\hline Blockage & 0 & $1 / 8$ & $2 / 8$ & $3 / 8$ \\
\hline & 1.38 & 1.31 & 1.22 & 1.12 \\
\hline
\end{tabular}

The CATIA software was used to carry out 3D solid modeling of the pump's inlet section, the impeller and the volute. The mesh of the impeller flow channel for different Plans was shown in Figure 1.

\section{Result analysis}

Centrifugal pump's prediction performance curves is shown as Fig 3. Pump's head was decreased as flow rate was increased. Pump's head and power shaft decreased as plugging rate of impeller channel increased in near design point. $P-Q$ curves of Plan B-D were lower than Plan A. Especially at high flow rate, extreme value of pump's $P-Q$ was appeared and presented non-overload characteristic. Fig. 3 indicated that pump's head and efficiency declined faster as plugging rate of impeller channel was increased. The error of different Plans' $\eta$ - $Q$ curve was close 
in near design point. However, the $\eta$ - $Q$ curve gradually moves down and becomes steeper at high flow rate condition.
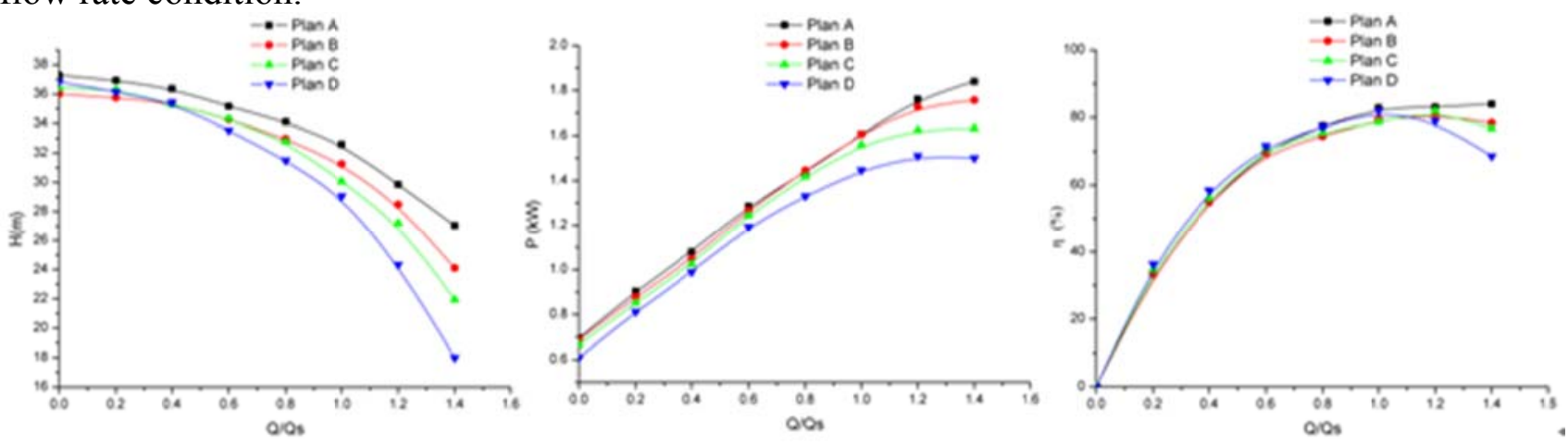

Fig. 3. The performance curves of pump with different Plans

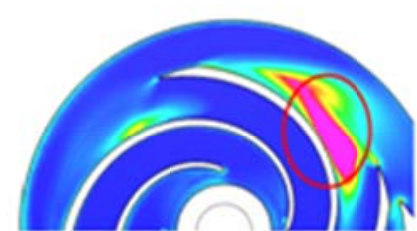

$0.4 \mathrm{Q}$ s (Plan A)

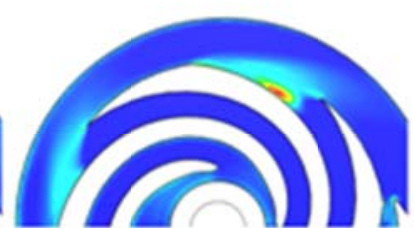

0.4 Qs (Plan D)

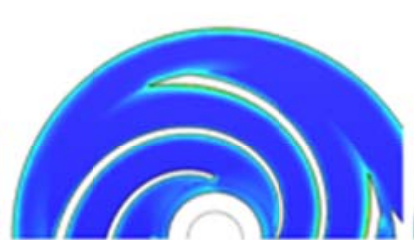

$1.2 \mathrm{Qs}(\operatorname{Plan} \mathrm{A})$

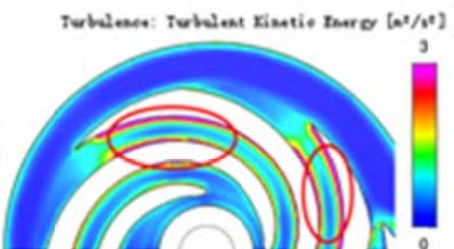

$1.2 \mathrm{Qs}($ Plan D)

Fig. 4. The impeller's turbulent kinetic energy distribution of different Plans.

Turbulent kinetic energy is a part of the energy that the fluid mechanical energy is converted into (dissipation) internal energy of fluid. The turbulent kinetic energy is greater and the flow stability is worse[4].

As shown in Fig 4, unstable areas were near the impeller channel which was closed to the tongue. In small flow condition, unstable area of Plan A was larger, the fluid of high turbulent kinetic energy was appeared, hydraulic loss was larger. At high flow rate condition, unstable area of Plan D was relatively large, which had a good explanation for that the efficiency of the Plan D in Fig 3 decreased sharply.

\section{Conclusions}

For low specific-speed centrifugal pump, the blockage of the impeller channel can reduce the turbulent kinetic energy in the impeller passage, the hydraulic loss was decreased and the efficiency of the pump was improved. At design condition, the efficiency of different Plans were similar. Adopting the method of blocking impeller passage can reduce effectively the pump's shaft power, and achieving pump's non-overload characteristics.

\section{References}

[1] Gianfranco Fagnani. The submersible pump-reliability in latest design[J]. World Pumps(1985): 00688866.

[2] Honghai C, Shouqi Y. The Optimal Designing Methods of Low-specific Pump[J]. Fluid Machinery, 2001(8) 19-23.

[3] Qu, Xiao, and Li Wang. Effects of Impeller Trimming Methods on Performances of Centrifugal Pump[J]. Journal of Energy Engineering (2016): 04016008.

[4] Davidson P. Turbulence: an introduction for scientists and engineers[M]. Oxford University Press, USA, 2015. 